\title{
Influence of Apical Periodontitis on Stress Oxidative Parameters in Diabetic Rats
}

\author{
Annelise Katrine Carrara Prieto, MSc, ${ }^{*}$ João Eduardo Gomes-Filbo, PbD, * \\ Mariane Maffei Azuma, MSc, ${ }^{*}$ Gustavo Sivieri-Araújo, PbD, * Luis Gustavo Narciso, MSc, ${ }^{\dagger}$ \\ Jucilene Conceição Souza, MSc, ${ }^{+}$Paulo César Ciarlini, PbD, ${ }^{\dagger}$ \\ and Luciano Tavares Angelo Cintra, $P b D^{*}$
}

\section{Ahstract}

Introduction: In this study, we investigated if apical periodontitis (AP) associated with diabetes influenced the levels of endogenous antioxidants, the total antioxidant capacity (TAC), and the oxidant parameter in the serum of Wistar rats. Methods: Forty male rats were divided into 4 equal groups: normal rats $(N)$, rats with AP $(A P)$, diabetic rats (D), and diabetic rats with AP $(D+A P)$. Diabetes was induced by alloxan $(150 \mathrm{mg} /$ $\mathrm{kg}$ ). AP was induced by exposing the pulpal tissue to the oral environment. After 36 days, blood and maxillae were collected. Albumin, bilirubin, uric acid, TAC, and malondialdehyde (MDA) levels were measured, and histologic analysis of the maxillae was performed. $P<.05$ was set as the threshold for statistical significance. Results: Uric acid levels were higher in the $D+A P$ group when compared with that of the N, D, and AP groups $(P<.05)$. The MDA concentration was higher in the $D$ and $D+A P$ groups when compared with the $N$ and AP groups $(P<.05)$. The level of albumin was lower in the $D+A P$ group when compared with the $N, A P$, and $D$ groups. Inflammatory infiltration was more intense in the periapical region in the $D+A P$ group compared with that in the AP group $(P<.05)$. Conclusions: Our findings indicate that diabetes may change the antioxidant status, increase the concentration of MDA and uric acid, and decrease albumin levels in the serum. In addition, AP can potentiate the effects of diabetes by reducing the levels of albumin and increasing the levels of uric acid. ( $J$ Endod 2017;43:1651-1656)

\section{Key Words}

Albumin, apical periodontitis, bilirubin, diabetes mellitus, oxidative stress, uric acid
$T^{\mathrm{h}}$ he bidirectional relationship between diabetes and apical periodontitis (AP) has been widely studied. Diabetes might influence the pathogenesis of AP by increasing the number of inflammatory cells (1) and contributing to the expansion of lesions $(1,2)$. In addition, AP can potentiate the systemic effects of diabetes by increasing the serum levels of triglycerides (3), creatinine (4), inflammatory cells (1), and proinflammatory cytokines such as interleukin 17 (5) and decreasing the glycemic control $(2,6)$. Considering that AP is an inflammatory process that causes tissue destruction (7), it is important to find newer strategies to decrease the inflammation. In this context, understanding the oxidative metabolism of the body is essential to unravel the mechanisms involved in tissue damage caused by diabetes as well as AP (8).

Oxidative stress occurs because of the deficiency of antioxidants or an increase in the production of free radicals (9). It is well-known that diabetes increases the oxidative stress, especially because hyperglycemia is directly related to the production of advanced glycation end products as well as reactive oxygen species (10). In overwhelming concentrations, reactive oxygen species can damage the cellular macromolecules, causing destruction of membranes, loss of cellular homeostasis, and, consequently, cell death (11). It may explain in part why AP is higher and more aggressive in diabetics compared with normoglycemic rats (5). In addition, our hypothesis is that the presence of AP may exacerbate oxidative stress systemically by increasing oxidative stress parameters, as observed in periodontal disease (12). Although AP and periodontal disease share important similarities with the microbial spectrum and the inflammatory response (13), until now, there was no study available in the literature relating the impact of AP on oxidative stress systemically. Thus, it would be interesting to investigate if AP can potentiate the production of antioxidants and free radicals in diabetic rats.

This understanding will also aid in the development of new therapies with an aim to modulate oxidative stress $(14,15)$. These therapies might help reduce the symptoms related to periapical lesions (16), periodontal disease (17), and diabetes $(18,19)$. Therefore, the aim of this study was to evaluate if AP alters the oxidative stress

From the *Department of Endodontics, School of Dentistry, São Paulo State University (Unesp) and ${ }^{\dagger}$ Department of Clinic and Surgery and Animal Reproduction, Veterinary Medicine, São Paulo State University (Unesp), Araçatuba, São Paulo, Brazil.

Address requests for reprints to Prof Luciano Tavares Angelo Cintra, Department of Endodontics, School of Dentistry, São Paulo State University (Unesp), Araçatuba, São Paulo, Brazil. E-mail address: lucianocintra@foa.unesp.br 0099-2399/\$ - see front matter

Copyright $\odot 2017$ American Association of Endodontists. http://dx.doi.org/10.1016/j.joen.2017.05.014 
parameters such as albumin, bilirubin, uric acid, the total antioxidant capacity (TAC), and malondialdehyde (MDA) in normoglycemic and diabetic rats.

\section{Experimental Design}

\section{Materials and Methods}

The study was approved by the Institutional Ethics Committee of Universidade Estadual Paulista, São Paulo, Brazil. Experiments were conducted in accordance with the relevant guidelines. Forty male Wistar rats (Rattus norvegicus albinus), 6 weeks old and weighing 200$250 \mathrm{~g}$, were used in this study. The animals were housed in a mini-isolator for rats (Alesco, São Paulo, Brazil) in temperaturecontrolled rooms and were given ad libitum access to water and food.

\section{Induction of Diabetes and AP}

The Wistar rats were divided into 4 groups of 10 rats each: normal (N), AP, diabetic (D), and diabetic with AP (D + AP). The rats were then administered anesthesia via intramuscular injections of ketamine $(87 \mathrm{mg} / \mathrm{kg}$, Francotar; Virbac do Brazil Ind e Com Ltda, Roseira, São Paulo, Brazil) and xylazine (13 mg/kg, Rompum, Bayer AS, São Paulo, Brazil). The rats were randomly assigned to receive intramuscular injections containing either citrate buffer solution (N and AP groups, $n=20$ ) or alloxan (D and D + AP groups, $n=20$ ) dissolved in a citrate buffer solution at $150 \mathrm{mg} / \mathrm{kg}$ body weight for the experimental induction of diabetes (day 0). After 6 days, all rats showed blood glucose levels more than $250 \mathrm{mg} / \mathrm{dL}$, which was the standard levels stablished for diabetic rats $(1,5,6)$.

To induce AP (day 6), surgical round burs (Drill LN Long Neck; Dentsply Ind e Com Ltda, Petrópolis, Brazil) were used to expose the pulp on the mesial surfaces of the right upper first molars to the oral environment (AP and D + AP groups) (1,3-5).

\section{Blood Sample Collection}

Thirty days after the induction of AP (day 36), the rats were anesthetized using a protocol described previously, and a cardiac puncture was performed to collect $5 \mathrm{~mL}$ blood from each rat. The blood samples were centrifuged to obtain plasma, which was stored at $-20^{\circ} \mathrm{C}$ and protected from light. Quantification of albumin, bilirubin, uric acid, TAC, and MDA was subsequently performed.

\section{Histologic Analyses}

After blood collection, the animals were killed with an overdose of the anesthetic solution. Maxillae were removed, postfixed in neutral buffered formalin for 48 hours, decalcified in buffered $(\mathrm{pH}=8)$ 17\% EDTA (Sigma-Aldrich, St Louis, M0), rinsed again in sterile water, dehydrated in ethanol, cleared in xylene, and embedded in paraffin. Serial slices (5- $\mu \mathrm{m}$ thickness) were prepared in the mesiodistal plane and stained with hematoxylin-eosin. The slices were examined sequentially under an optical microscope (DM 4000 B; Leica Microsystems, Wetzlar, Germany).

Analyses were performed by a single calibrated operator who was blind to the specimen's group affiliation. The inflammatory infiltrate was evaluated for intensity and extension. The average number of cells per field and the extension that went beyond either of the apical foramen (AP groups) were considered. For each experimental group, the number of cells was calculated as the average of 10 separate areas $(\times 400$ magnification). The intensity of the inflammatory infiltrate was graded as absent ( 0 to few inflammatory cells: score 1$)$, mild ( $<25$ cells: score 2 ), moderate (25-125 cells: score 3 ), and severe (>125 cells: score 4). The extension of the inflammatory infiltrate was graded as absent ( 0 to few inflammatory cells: score 1 ), mild (cells occupying up to a 300- $\mu \mathrm{m}$ length: score 2), moderate (cells occupying up to a $600-\mu \mathrm{m}$ length: score 3), and severe (cells occupying $>\mathrm{a}$ 600- $\mu \mathrm{m}$ length: score 4).

For the AP and D + AP groups, the area of the periapical lesion was measured histometrically. For each rat, 5 serial histologic sections were analyzed by an image processing system that consisted of a light microscope (DM 4000 B, Leica), a color camera (DFC 500, Leica Microsystems), a color image processor (Leica Qwin V3 software, Leica Microsystems), and a personal computer (Intel Corel 5 [Intel, Santa Clara, CA] with Windows 10 [Microsoft, Redmond, WA]).

\section{Measurement of Oxidative Stress}

All the biochemical tests were performed with the use of commercial reagents (BioSystems, Barcelona, Spain) at $37^{\circ} \mathrm{C}$ in an automated analyzer (BTS-370 Plus, BioSystems), which had been previously calibrated. The blood concentration of albumin was determined by the bromocresol green assay, bilirubin by using diazotized sulfanilic acid, and uric acid by the uricase/peroxidase enzymatic assay.

The serum level of TAC was quantified in an automated analyzer by inhibiting $2.2^{\prime}$-azino diethylbenzothiazoline sulfonic acid cation formation. TAC analyses were monitored with a standard antioxidant specific for automation.

Blood lipid peroxidation was determined by quantifying the plasma thiobarbituric acid reactive substances. Absorbance at $530 \mathrm{~nm}$ was measured in a plate reader (Spectra Count Reader; Packard BioScience, Meriden, CT) in accordance with the manufacturer's recommendations, starting with a standard commercial MDA solution (500 mmol/L) and using a computer program (GraphPad Prism, version 4; GraphPad Software, San Diego, CA). A curve for final concentrations of $0,0.625,1.25,2.5,5,10,25$, and $50 \mathrm{nmol} / \mathrm{mL} \mathrm{MDA}$ was developed. Each point on the curve was obtained from the mean value of 10 repetitions.

\section{Statistical Analyses}

The values obtained in parametric tests were tabulated and presented as the means observed in each group. Statistical differences between groups were determined by analysis of variance followed by the

TABLE 1. Weight (g) and Blood Glucose (mg/dL) in Rats from All Groups (Mean and Standard Deviation [SD*])

\begin{tabular}{|c|c|c|c|c|c|}
\hline \multirow[b]{2}{*}{ Groups } & \multicolumn{2}{|c|}{ Weight $(g) \pm S D *$} & \multicolumn{3}{|c|}{ Blood glucose $(\mathrm{mg} / \mathrm{dL}) \pm S D^{*}$} \\
\hline & Day 0 & Day 36 & Day 0 & Day 6 & Day 36 \\
\hline $\mathrm{N}$ & $269.8 \pm 20.7^{a}$ & $410.0 \pm 31.1^{a}$ & $73.7 \pm 9.8^{a}$ & $79.8 \pm 11.1^{a}$ & $95.0 \pm 7.3^{a}$ \\
\hline AP & $268.1 \pm 23.6^{a}$ & $381.1 \pm 31.5^{a}$ & $79.8 \pm 9.1^{a}$ & $82.8 \pm 9.0^{a}$ & $89.0 \pm 8.8^{a}$ \\
\hline $\mathrm{D}$ & $273.5 \pm 11.7^{a}$ & $210.0 \pm 38.3^{b}$ & $73.5 \pm 12.3^{a}$ & $597.3 \pm 6.3^{b}$ & $586.0 \pm 21.0^{b}$ \\
\hline$D+A P$ & $260.0 \pm 24.2^{a}$ & $211.4 \pm 30.5^{b}$ & $74.4 \pm 7.9^{a}$ & $555.4 \pm 51.9^{c}$ & $600.0 \pm 0.0^{\mathrm{b}}$ \\
\hline
\end{tabular}

AP, apical periodontitis; D, diabetic; D + AP, diabetic with apical periodontitis; N, normal.

*Different letters in the columns indicate statistical differences, $P<.05$. 
Tukey test for multiple comparisons. The histologic analysis scores were analyzed using the Mann-Whitney $U$ test $(P<.05)$. All statistical tests were performed using the software Pacotico (Visual FoxPro, Microsoft).

\section{Results}

At the beginning of the experiment, the groups had a similar mean body weight $(P>.05)$. After 36 days, diabetic groups (D and D + AP) presented with reduced body weight compared with the normoglycemic groups $(P<.05)$. Blood glucose examined on day 0 revealed normoglycemic condition at the beginning of the experiment in all groups.
After 6 days, the diabetic groups presented with a significant increase of blood glucose $(P<.05)$. On the last experimental day (day 36), groups of diabetic rats ( $\mathrm{D}$ and $\mathrm{D}+\mathrm{AP}$ ) were confirmed to be hyperglycemic (Table 1). The periapical regions in $\mathrm{N}$ or $\mathrm{D}$ rats did not show evidence of inflammation (Fig. $1 A-H$ ). Diabetes alone was not found to produce significant changes during the period studied. In the AP and D + AP groups, the pulp showed total necrosis 30 days after exposure. Periapical lesions were established, were restricted exclusively to the periapical region, and showed characteristics of periapical granuloma (Fig. 1). Rats in the AP group had obvious lesions that were composed predominantly of a chronic inflammatory infiltrate graded as moderate,

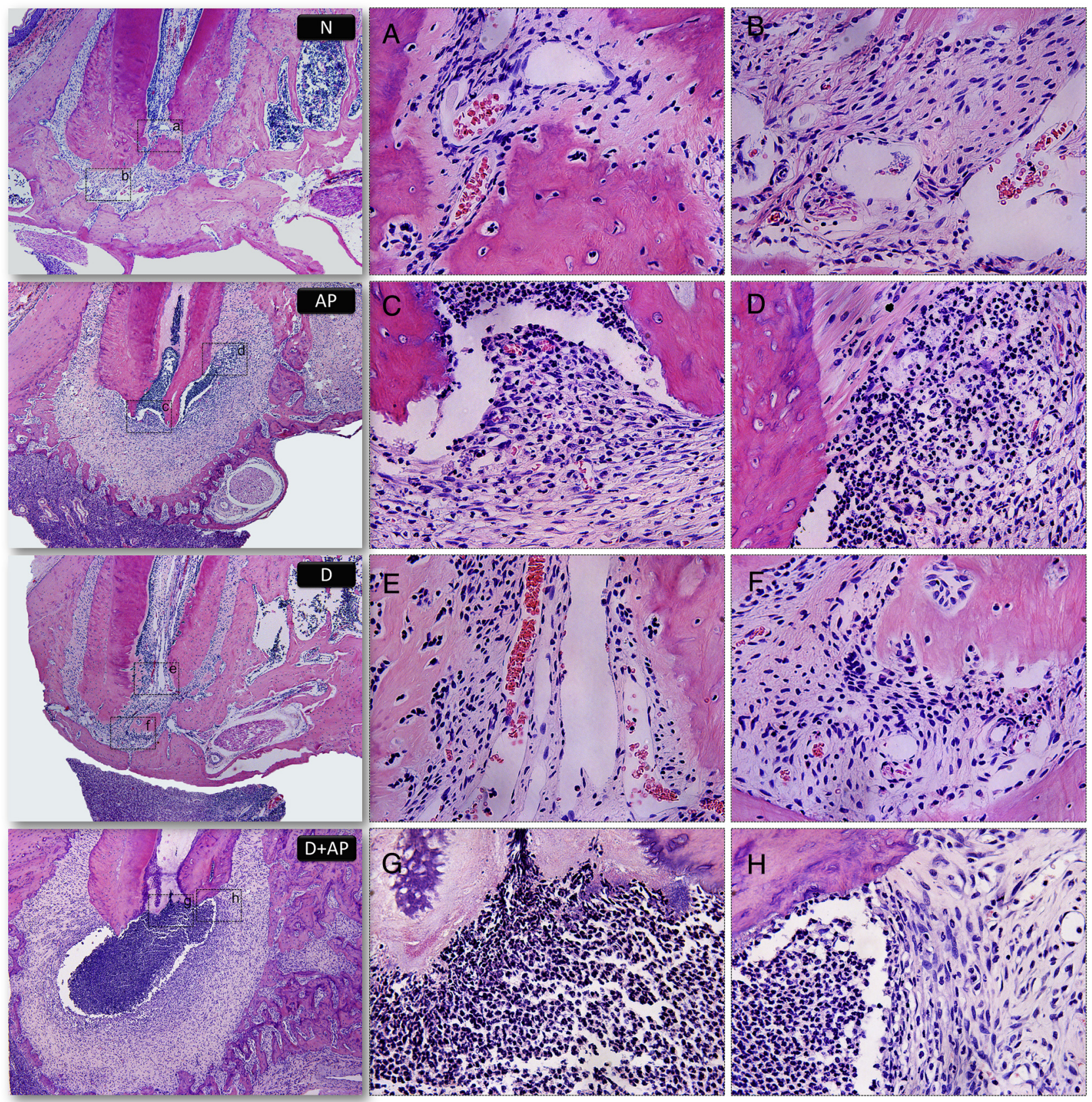

Figure 1. Histologic findings 36 days after the induction of diabetes and 30 days after the induction of AP. $(A$ and $B)$ The pulp, apical, and periapical tissues are free of inflammatory cell infiltrates in the $\mathrm{N}$ group. $(C$ and $D$ ) Pulp necrosis, moderate chronic inflammatory cell infiltration in the periapical region, and bone resorption in the AP group. ( $E$ and F) The normal pulp, apical and periapical tissues seen in the D group. $(G$ and $H$ ) Pulp necrosis, intense acute inflammatory cell concentrations near the tooth apex region, and large bone resorption in the D + AP group. Hematoxylin-eosin staining, $\times 50$ and $\times 400$ original magnification. 
TABLE 2. Scores, Median, Mean, and Standard Deviation (SD) of Histologic Findings in the Rats from All Groups

\begin{tabular}{|c|c|c|c|c|c|}
\hline \multirow[b]{2}{*}{ Histologic Parameters } & \multirow[b]{2}{*}{ Scores } & \multicolumn{4}{|c|}{ Groups } \\
\hline & & $\mathbf{N}$ & AP & D & $D+A P$ \\
\hline \multirow[t]{5}{*}{ Inflammatory infiltrate } & 1 & $10 / 10$ & $0 / 10$ & $10 / 10$ & $0 / 10$ \\
\hline & 2 & $0 / 10$ & $1 / 10$ & $0 / 10$ & $0 / 10$ \\
\hline & 3 & $0 / 10$ & $7 / 10$ & $0 / 10$ & $3 / 10$ \\
\hline & 4 & $0 / 10$ & $2 / 10$ & $0 / 10$ & $7 / 10$ \\
\hline & Median* & 1 & $3^{a}$ & 1 & $4^{b}$ \\
\hline \multirow{5}{*}{ Inflammation extent } & 1 & $10 / 10$ & $0 / 10$ & $10 / 10$ & $0 / 10$ \\
\hline & 2 & $0 / 10$ & $3 / 10$ & $0 / 10$ & $0 / 10$ \\
\hline & 3 & $0 / 10$ & $5 / 10$ & $0 / 10$ & $2 / 10$ \\
\hline & 4 & $0 / 10$ & $2 / 10$ & $0 / 10$ & $8 / 10$ \\
\hline & Median* & 1 & $3^{\mathrm{a}}$ & 1 & $4^{\mathrm{b}}$ \\
\hline $\begin{array}{l}\text { Periapical lesions } \\
\quad\left(\times 10.000 \mu \mathrm{m}^{2}\right)\end{array}$ & Mean $\pm S D^{*}$ & $12.88 \pm 1.63^{\mathrm{a}}$ & $75,4 \pm 11.8^{b}$ & $2.56 \pm 1.61^{a}$ & $93.6 \pm 16.4^{c}$ \\
\hline
\end{tabular}

AP, apical periodontitis; D, diabetic; D + AP, diabetic with apical periodontitis; N, normal.

*Different letters on the line indicate statistical differences, $P<.05$

consisting primarily of neutrophils (polymorph nuclear cells) and mononuclear cells (Fig. 1). Furthermore, cement and bone resorption was observed in the AP and D + AP groups in the presence of dentin (Fig. 1). Moreover, in the D + AP group, there was intense periapical inflammatory infiltrates that were more severe than the AP group $(P<.05)$. Histometrically, the D + AP group exhibited the most significant periapical lesions (Table 2).

\section{Antioxidant Parameters}

The blood albumin concentration in rats in the diabetic groups (D and $\mathrm{D}+\mathrm{AP}$ ) was lower than that in the normoglycemic groups ( $\mathrm{N}$ and AP) $(P<.05)$. Moreover, the periapical lesion decreased the albumin levels $(P<.05)$. There was no statistically significant difference between the diabetic and normoglycemic groups with respect to bilirubin $(P>.05)$ despite the slight decrease in the diabetic groups. The level of uric acid was higher in the diabetic groups (D and D $+A P)$ when compared with that in the normoglycemic groups (N and AP) $(P<.05)$. Furthermore, among the diabetic groups, the group $\mathrm{D}+$ AP had higher uric acid compared with group D (Table 3) $(P<.05)$. There was no statistically significant difference in TAC level despite the slight increase in the $\mathrm{D}+\mathrm{AP}$ group $(P>.05)$. In diabetic rats, there was an increase in the concentration of MDA regardless of the presence of AP $(P<.05)$.

\section{Discussion}

Wistar rats were selected for the study because of anatomic similarity of their pulp and periapical tissues with humans. All the rats showed similar weight and blood glucose levels at the beginning of the study. Alloxan-induced diabetes in the animal model presented with symptoms similar to those found in humans with diabetes, such as weight loss, polydipsia, polyuria, and hyperglycemia (20). However, the present model has some limitations, including the fast development of diabetes in young animals who did not receive any treatment. The results and observations of the present study should not be directly compared with humans. On the other hand, it may be considered the first step to obtaining evidence to study patients who may be receiving treatment for glycemic control and with normal development of diabetes.

Diabetic rats (groups D and D + AP) showed a decrease in their body weight during the experimental period, which was statistically significant compared with normal rats (groups $\mathrm{N}$ and $\mathrm{AP}$ ). This difference in body weight is caused by the absence of insulin metabolism leading to a catabolic state that causes severe depletion of energy stores and proteins, particularly those found in the skeletal muscle (21). The changes in body weight were observed in another study and even changes in organs such as the liver, brain, heart, and gonads (22).

The diabetic rats had higher blood glucose levels when compared with the normoglycemic rats, indicating that hyperglycemia persisted in the diabetic rats. In addition, it was found that the presence of a single periapical lesion did not influence the blood glucose levels in diabetic rats during the 30 days of study. However, a previous study had shown that AP influenced the glucose levels in diabetic rats when it was associated with periodontal disease (6). It probably happened because the mechanism in which chronic oral diseases alter metabolic control in diabetic rats involves the activation of a proinflammatory pathway (6). Thus, when AP was associated with periodontal disease in diabetic rats, the inflammatory response, including activation of proinflammatory pathways, might be higher when compared with AP alone.

Microscopic examination revealed no inflammation in the $\mathrm{N}$ and $\mathrm{D}$ groups. Thus, the presence of diabetes did not induce inflammation of the pulp and periapical tissues during the study period (36 days). However, in the groups with AP (AP and D + AP), an increase in the intensity and extent of the inflammatory infiltrate was noted in the presence of

TABLE 3. Endogenous Antioxidants (mg/dL), Total Antioxidant Capacity (TAC) (mmol/L), and Malondialdehyde (MDA) ( $\mu$ mol/L) in the Serum of Rats from All the Groups (Mean and Standard Deviation [SD])

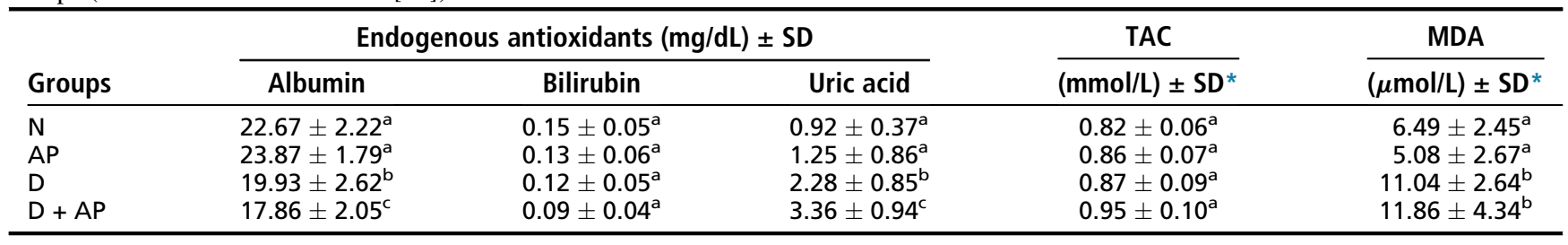

AP, apical periodontitis; D, diabetic; D + AP, diabetic with apical periodontitis; N, normal.

*Different letters on the column indicate statistical differences, $P>.05$. 
diabetes. These findings are consistent with other studies that have evaluated pulpal infection associated with diabetes $(1,2)$. Thus, our study confirms that the progression of diabetes has an effect on oral tissue inflammation.

Periapical lesions in the diabetic rats were higher and more aggressive compared with that in normoglycemic rats $(1,5)$. Diabetes exacerbates the process of oral tissue inflammation by compromising tissue integrity. Increased aggressiveness of periapical lesions in diabetics is caused by the hyperglycemic condition that interferes with the function of macrophages, pulp blood circulation (23), growth and differentiation of osteoblasts (24), bone homeostasis (25), and increased bacterial load (26).

The level of albumin, an endogenous antioxidant, was decreased in the diabetic rats (groups D and D $+\mathrm{AP}$ ) when compared with the normoglycemic rats (groups $\mathrm{N}$ and AP). The decrease in albumin levels may be explained by the hyperglycemia caused by diabetes. Hyperglycemia is responsible for triggering various events such as the formation of advanced glycation end products, reactive oxygen species, activation of protein kinase $\mathrm{C}$, and growth factors that eventually lead to renal injury (27). Renal injury because of hyperglycemia causes diabetic nephropathy (28). The loss of albumin in urine, which leads to hypoalbumine$\mathrm{mia}$, is used to assess the nutritional and/or inflammatory condition of a patient with chronic kidney disease (29). Our results showed that AP was able to alter the concentration of the albumin in the serum of diabetic rats after 30 days, suggesting that AP might potentiate the systemic effects of diabetes. These effects, especially diabetic nephropathy, occur rapidly in the rat model (30) and differ from the reality of the development of diabetes in humans (19).

Serum levels of the antioxidant uric acid were significantly higher in the diabetic rats (D and D + AP groups) compared with the normoglycemic rats (N and AP groups). Diabetes leads to nephropathy and subsequently chronic kidney disease, and uric acid serves as a marker of chronic kidney disease (31). This explains the hyperuricemia in diabetic rats. The increase in uric acid level in the D + AP group compared with that in the D group also suggests that AP potentiates diabetes.

Thus, AP not only decreased the endogenous antioxidant albumin but also increased the antioxidant uric acid in diabetic rats. These results are very significant because it suggests that AP must be a concern for diabetic individuals because it potentiated the effects of diabetes in renal tissues, as observed through the alteration of albumin and uric acid, which are important markers for chronic kidney disease. This extreme situation of the animal model of diabetes has no direct translation to patients. Thus, further investigations should be performed, especially in humans, to confirm the bidirectional relationship between diabetes and AP on oxidative parameters.

Bilirubin and TAC levels were not significantly different among the groups. There are no studies investigating the relationship between bilirubin and AP. However, a study that assessed the association between bilirubin levels and periodontal disease has suggested that bilirubin is involved in the inhibition of inflammatory pathways during the development of periodontal disease (5). There are no studies evaluating the association between blood TAC, AP, and diabetes. However, it has been reported previously that an increase in TAC levels in the periapical tissues occurs in periapical periodontitis (32). In addition, it has been observed that an increase in the serum TAC levels occurs in the presence of periodontal disease (33) in the absence of diabetes.

In this study, MDA was increased in the diabetic groups (D and D + AP). Hyperglycemia might have induced the formation of free radicals, including the hydroxyl radical that captured the hydrogen from the plasma membrane's lipids, thus promoting lipoperoxidation (34).
However, AP did not alter the MDA levels in the blood. However, studies have shown an increase in the end products of lipid peroxidation in AP tissue and their erythrocytes, revealing that AP can influence systemic health (35).

The exposed findings are very important because they suggested that the treatment and management of AP may improve the systemic oxidative condition induced by diabetes. In addition, the results instigate the development of future studies to confirm if the endodontic treatment can reduce the oxidative stress in diabetic individuals.

Based on the findings of this study, it can be concluded that diabetes changed the antioxidant status by increasing the concentration of MDA and uric acid and decreasing the serum levels of albumin. In addition, AP associated with diabetes reduced the serum levels of albumin and increased the endogenous antioxidant uric acid, suggesting that AP potentiates the systemic effects of diabetes.

\section{Acknowledgments}

\author{
Supported by FAPESP (grant no. 2012/04588-0). \\ The authors deny any conflicts of interest related to this study.
}

\section{References}

1. Cintra LT, Da Silva Facundo AC, Prieto AK, et al. Blood profile and histology in oral infections associated with diabetes. J Endod 2014;40:1139-44.

2. Iwama A, Nishigaki N, Nakamura K, et al. The effect of high sugar intake on the development of periradicular lesions in rats with type 2 diabetes. J Dent Res 2003;82:322-5.

3. Cintra LT, da Silva Facundo AC, Azuma MM, et al. Pulpal and periodontal diseases increase triglyceride levels in diabetic rats. Clin Oral Investig 2013;17:1595-9.

4. Cintra LT, da Silva Facundo AC, Valentim D, et al. Effect of oral infections on serum creatinine levels in diabetic rats. Int J Diabetol Vasc Dis Res 2013;1:1-6.

5. Cintra LT, Samuel R0, Azuma MM, et al. Apical periodontitis and periodontal disease increase serum IL-17 levels in normoglycemic and diabetic rats. Clin Oral Investig 2014;18:2123-8.

6. Cintra LT, Samuel RO, Facundo AC, et al. Relationships between oral infections and blood glucose concentrations or HbA1c levels in normal and diabetic rats. Int Endod J 2014; 47:228-37.

7. Kawashima N, Okiji T, Kosaka T, et al. Kinetics of macrophages and lymphoid cells during the development of experimentally induced periapical lesions in rat molars: a quantitative immunohistochemical study. J Endod 1996;22:311-6.

8. Chapple IL, Milward MR, Dietrich T. The prevalence of inflammatory periodontitis is negatively associated with serum antioxidant concentrations. J Nutr 2007;137: 657-64.

9. Halliwell B, Gutteridge JM. Free Radicals in Biology and Medicine, 4th ed. Oxford, UK: Clarendon; 2007.

10. Fierro JA. Nefropatía diabética: fisiopatologia, diagnóstico, evolución clínica y epidemiologia. Rev Med Clin Condes 2009;20:639-50.

11. Patil VS, Patil VP, Gokhale N, et al. Chronic periodontitis in type 2 diabetes mellitus: oxidative stress a common factor in periodontal tissue injury. J Clin Diagn Res 2016; $10: \mathrm{BC} 12-6$.

12. Ambati M, Rani KR, Reddy PV, et al. Evaluation of oxidative stress in chronic periodontitis patients following systemic antioxidant supplementation: a clinical and biochemical study. J Nat Sci Biol Med 2017;8:99-103.

13. Rupfi S, Kannengiesser S, Merte K, et al. Comparison of profiles of key periodontal pathogens in periodontium and endodontium. Endod Dent Traumatol 2000;16: 269-75.

14. Gao X, Xu Y, Xu B, et al. Allopurinol attenuates left ventricular dysfunction in rats with early stage of streptozotocin-induced diabetes. Diabetes Metab Res Rev 2012:28:409-17.

15. Kim KJ, Yoon KY, Hong HD, et al. Schisandra chinensis prevents hepatic lipid peroxidation and oxidative stress in rats subjected to heat environmental stress. Phytother Res 2012;26:1674-80.

16. Wolle CF, de Aguiar Zollmann L, de Aguiar Zollmann L, et al. Effects of the antioxidant agent tempol on periapical lesions in rats with doxorubicin-induced cardiomyopathy. J Endod 2012;38:191-5.

17. Tilakaratne A, Soory M. Osteoblastic responses to LPS, glucose-oxidised LDL and minocycline: therapeutic targets for periodontal and cardiometabolic diseases. Recent Pat Endocr Metab Immune Drug Discov 2012;6:73-84.

18. Adam SH, Giribabu N, Kassim N, et al. Protective effect of aqueous seed extract of Vitis Vinifera against oxidative stress, inflammation and apoptosis in the pancreas of adult male rats with diabetes mellitus. Biomed Pharmacother 2016;81:439-52. 
19. Chou ST, Tseng ST. Oxidative stress markers in type 2 diabetes patients with diabetic nephropathy. Clin Exp Nephrol 2017;21:283-92.

20. Ige AO, Adewoye EO, Okwundu $\mathrm{NC}$, et al. Oral magnesium reduces gastric mucosa susceptibility to injury in experimental diabetes mellitus. Pathophysiology 2016;23:87-93

21. Hebert SI, Nair KS. Protein and energy metabolism in type 1 diabetes. Clin Nutr 2010;29:13-7.

22. Cintra LT, Samuel RO, Prieto AK, et al. Oral health, diabetes, and body weight. Arch Oral Biol 2017;73:94-9.

23. Bender IB, Bender AB. Diabetes mellitus and the dental pulp. J Endod 2003;29: 383-9.

24. Zayzafoon M, Stell C, Irwin R, et al. Extracellular glucose influences osteoblast differentiation and c-Jun expression. J Cell Biochem 2000;79:301-10.

25. Mathieu C, Gysemans C, Giulietti A, et al. Vitamin D and diabetes. Diabetologia 2005: 48:1247-57.

26. Iwama A, Morimoto T, Tsuji M, et al. Increased number of anaerobic bacteria in the infected root canal in type 2 diabetic rats. Oral Surg Oral Med Oral Pathol Oral Radiol Endod 2006;101:681-6.

27. Mogensen CE, Christensen CK. Predicting diabetic nephropathy in insulin-dependent patients. N Engl J Med 1984;311:89-93.

28. Menon V, Greene T, Wang X, et al. C-reactive protein and albumin as predictors of all-cause and cardiovascular mortality in chronic kidney disease. Kidney Int 2005; 68:766-72.
29. Nakagawa T, Kang DH, Feig D, et al. Unearthing uric acid: an ancient factor with recently found significance in renal and cardiovascular disease. Kidney Int 2006; 69:1722-5.

30. Borgohain MP, Lahkar M, Ahmed S, et al. Small molecule inhibiting nuclear factor$\mathrm{kB}$ ameliorates oxidative stress and suppresses renal inflammation in early stage of alloxan-induced diabetic nephropathy in rat. Basic Clin Pharmacol Toxicol 2017; 120:442-9.

31. Dezerega A, Madrid S, Mundi V, et al. Pro-oxidant status and matrix metalloproteinases in apical lesions and gingival crevicular fluid as potential biomarkers for asymptomatic apical periodontitis and endodontic treatment response. J Inflamm (Lond) 2012;9:8.

32. Žilinskas J, Kubilius R, Žekonis G, et al. Total antioxidant capacity of venous blood, blood plasma, and serum of patients with periodontitis, and the effect of Traumeel S on these characteristics. Medicina (Kaunas) 2011;47:193-9.

33. Brunzell IB, Chait AJ. Reduced plasma peroxyl radical trapping capacity and increased susceptibility of LDL to oxidation in poorly controlled IDDM. Diabetes 1994; 43:1010-4.

34. Cherkashin SI. Lipid peroxidation in persons with chronic odontogenic foci of infection. Stomatologiia (Mosk) 1989;68:15-7.

35. Marton IJ, Balla G, Hegedus C, et al. The role of reactive oxygen intermediates in the pathogenesis of chronic apical periodontitis. Oral Microbiol Immunol 1993;8: $254-7$. 